\title{
Protein synthesis inhibitors stimulate MondoA transcriptional activity by driving an accumulation of glucose 6-phosphate
}

\author{
Blake R. Wilde ${ }^{1,2}$, Mohan R. Kaadige ${ }^{1,3}$, Katrin P. Guillen ${ }^{4}$, Andrew Butterfield ${ }^{4}$, Bryan E. Welm ${ }^{4}$ and Donald E. Ayer ${ }^{*}$
}

\begin{abstract}
Background: Protein synthesis is regulated by the availability of amino acids, the engagement of growth factor signaling pathways, and adenosine triphosphate (ATP) levels sufficient to support translation. Crosstalk between these inputs is extensive, yet other regulatory mechanisms remain to be characterized. For example, the translation initiation inhibitor rocaglamide A (RocA) induces thioredoxin-interacting protein (TXNIP). TXNIP is a negative regulator of glucose uptake; thus, its induction by RocA links translation to the availability of glucose. MondoA is the principal regulator of glucose-induced transcription, and its activity is triggered by the glycolytic intermediate, glucose 6-phosphate (G6P). MondoA responds to G6P generated by cytoplasmic glucose and mitochondrial ATP (mtATP), suggesting a critical role in the cellular response to these energy sources. TXNIP expression is entirely dependent on MondoA; therefore, we investigated how protein synthesis inhibitors impact its transcriptional activity.

Methods: We investigated how translation regulates MondoA activity using cell line models and loss-of-function approaches. We examined how protein synthesis inhibitors effect gene expression and metabolism using RNAsequencing and metabolomics, respectively. The biological impact of RocA was evaluated using cell lines and patient-derived xenograft organoid (PDxO) models.
\end{abstract}

Results: We discovered that multiple protein synthesis inhibitors, including RocA, increase TXNIP expression in a manner that depends on MondoA, a functional electron transport chain and mtATP synthesis. Furthermore, RocA and cycloheximide increase mtATP and G6P levels, respectively, and TXNIP induction depends on interactions between the voltage-dependent anion channel (VDAC) and hexokinase (HK), which generates G6P. RocA treatment impacts the regulation of $\sim 1200$ genes, and $\sim 250$ of those genes are MondoA-dependent. RocA treatment is cytotoxic to triple negative breast cancer (TNBC) cell lines and shows preferential cytotoxicity against estrogen receptor negative (ER-) PDxO breast cancer models. Finally, RocA-driven cytotoxicity is partially dependent on MondoA or TXNIP.

Conclusions: Our data suggest that protein synthesis inhibitors rewire metabolism, resulting in an increase in mtATP and G6P, the latter driving MondoA-dependent transcriptional activity. Further, MondoA is a critical component of the cellular transcriptional response to RocA. Our functional assays suggest that RocA or similar translation inhibitors may show efficacy against ER- breast tumors and that the levels of MondoA and TXNIP should be considered when exploring these potential treatment options.

\footnotetext{
* Correspondence: don.ayer@hci.utah.edu

'Department of Oncological Sciences, Huntsman Cancer Institute, University of Utah, Salt Lake City, UT 84112, USA

Full list of author information is available at the end of the article
}

\section{$\triangle B M C$}

C C The Author(s). 2020 Open Access This article is licensed under a Creative Commons Attribution 4.0 International License, which permits use, sharing, adaptation, distribution and reproduction in any medium or format, as long as you give appropriate credit to the original author(s) and the source, provide a link to the Creative Commons licence, and indicate if changes were made. The images or other third party material in this article are included in the article's Creative Commons licence, unless indicated otherwise in a credit line to the material. If material is not included in the article's Creative Commons licence and your intended use is not permitted by statutory regulation or exceeds the permitted use, you will need to obtain permission directly from the copyright holder. To view a copy of this licence, visit http://creativecommons.org/licenses/by/4.0/ The Creative Commons Public Domain Dedication waiver (http://creativecommons.org/publicdomain/zero/1.0/) applies to the data made available in this article, unless otherwise stated in a credit line to the data. 


\section{Background}

A unifying characteristic of oncogenes is their ability to drive anabolic metabolism to support the biosynthesis of macromolecules. Oncogenes also impose significant metabolic stress on cells [1]. For example, as a result of increased protein synthesis, cancer cells experience depletion of local nutrients [2], which can lead to accumulation of reactive oxygen species (ROS) and other metabolic challenges that if unchecked result in cell death [3]. These findings suggest that cells must integrate information about translation rate with the pathways that control nutrient availability. Recently, protein synthesis inhibitors have received attention as potential anticancer therapeutics [4-7], with translation initiation inhibitors among the most promising candidates. The full mechanistic and biological consequences of targeting translation initiation have not been described.

The translation inhibitor RocA induces expression of TXNIP in a number of cell types [6]; however, the underlying mechanisms were not explored. TXNIP has pleiotropic function $[8,9]$, including acting as a very potent negative regulator of glucose uptake [10]. Therefore, TXNIP may bridge translation initiation or elongation rate to the availability of glucose. TXNIP expression is strongly, if not entirely dependent on the MondoA transcription factor and glucose $[10,11]$. Mechanistically, glucose 6-phosphate (G6P) drives translocation of MondoA from the outer mitochondrial membrane (OMM) to the nucleus where it binds the promoters of its target genes and recruits cofactors that initiate transcription $[10,12,13]$. MondoA binds a double Ebox carbohydrate response element (ChoRE) in the TXNIP promoter to drive its expression in response to elevated glucose levels $[11,14,15]$.

In addition to an absolute functional requirement on glucose $[10,11]$, we recently showed that MondoA transcriptional activity is also highly dependent on mtATP [13]. Our data suggest that MondoA functions as a coincidence detector, only being active when above threshold levels of glucose and mtATP are available to generate enough G6P to drive MondoA activity [13]. Collectively, our data suggest that MondoA is a sensor of high cellular energy charge exemplified by its two most prevalent nutrient sources and is critical for the adaptive transcriptional response to a hyper-nutrient state.

Here, we investigate whether MondoA is required for protein synthesis inhibitors to increase TXNIP expression. We provide evidence that protein synthesis inhibitors cause metabolic rewiring, resulting in increased levels of mtATP and G6P that drive MondoA transcriptional activity. Further, the cytotoxic effect of RocA depends on both MondoA and TXNIP, suggesting that they may be critically required for the utility of protein synthesis inhibitors in clinical settings.

\section{Methods}

\section{Cell culture}

All cell lines were maintained at $37^{\circ} \mathrm{C}$ in $5 \% \mathrm{CO}_{2}$. Dulbeccos Minimal Essential Media (DMEM) with penicillin/streptomycin and 10\% fetal bovine serum (FBS) (Gibco) was used for murine embryonic fibroblasts (MEFs), HeLa, MDA-MB-231, L6, C2C12, and 293T (all from ATCC) and MDA-MB-157 cells (a gift from Andrea Bild, University of Utah). $\mathrm{TSC}^{-1-}$ and $\mathrm{TSC}^{-1-}$ :hTSC2 MEFs were a gift of Brendan Manning, Harvard University. MondoA ${ }^{-1-}$ MEFs were created from day 15 embryos as described previously [16].

\section{Plasmids}

pcDNA3.1-MondoA-V5, pcDNA3-Mlx-FLAG, and LXSHMondoA as well as TXNIP promoter luciferase reporter plasmids (wild type and ChoRE mutant) have been described [12, 16]. pcDNA3-Mit-ATEAM (pcDNA3mitAT1.03) was a gift of Hiroyuki Noji, Rikkyo University [17]. pLKO.1-shScrm and pLKO.1-shTXNIP were obtained from Sigma Aldrich. Standard molecular cloning techniques were used to generate pLVX-TetOne-PuroMYC(T58A). The pLVX-TetOne-Puro vector was obtained from Clontech Laboratories. Transfections were performed using Lipofectamine 2000 (Thermo Fisher) or Lipofectamine 3000 (Thermo Fisher).

\section{Protein synthesis inhibitor treatments}

Growth media was replaced with glucose-free DMEM with penicillin/streptomycin and 10\% FBS for $6 \mathrm{~h}$. Media was then replaced with glucose-containing DMEM with penicillin/streptomycin, 10\% FBS, and translation inhibitors for $16 \mathrm{~h}$. Unless otherwise indicated, the protein synthesis inhibitors were added at the following concentrations: cycloheximide (CHX) (Sigma Aldrich), $50 \mu \mathrm{g} /$ $\mathrm{ml}$; emetine (Sigma Aldrich), $100 \mu \mathrm{g} / \mathrm{ml}$; puromycin (Puro) (Sigma Aldrich), $100 \mu \mathrm{g} / \mathrm{ml}$; and rocaglamide A (Santa Cruz), 25-100 nM. Dialyzed FBS was prepared by dialysis 3 times against 40 -fold excess water to remove small molecules.

\section{Quantitative real-time PCR}

Total RNA was extracted using the RNAeasy Kit (Qiagen). cDNA was synthesized from 0.1 to $1 \mu \mathrm{g}$ RNA using GoScript reverse transcription kit (Promega). qPCR was performed using OneTaq Hot Start DNA Polymerase [18], SYBR/ROX Combo PCR DNA Fluorescence Dye (Thermo Fisher), and dNTPs (Thermo Fisher). The $\Delta \Delta \mathrm{Ct}$ method with normalization to actin levels was used to analyze the data. Three biological replicates were used to determine mRNA levels and calculate significance. Three technical replicates were performed for every biological sample. TXNIP primers: forward-TGACTTTGGCCTACAGTGGG and reverse-TTGCGCTTCTCCAGATACTGC; Actin primers: 
forward-TCCATCATGAAGTGTGACGT and reverseTACTCCTGCTTGCTGATCCAC.

\section{Immunofluorescence}

Cells were transfected with plasmids containing MondoAV5 and FLAG-Mlx using Lipofectamine 2000 (Thermo Fisher). Following protein synthesis inhibitor treatment, cells were fixed on glass coverslips using ice-cold 100\% methanol for $15 \mathrm{~min}$ and stained using standard immunofluorescence procedures. Mouse anti-V5 (Thermo Fisher) antibody was used at 1:2000; rabbit anti-FLAG (Cell Signaling) antibody was used at 1:2000.

\section{Metabolomics}

Gas chromatograph-mass spectrometry (GC-MS) was used to determine metabolite levels as described previously [13]. Metabolites were harvested from cells using 90\% methanol and analyzed over a $30 \mathrm{~m}$ Phenomex ZB5-5 MSi column. Data was analyzed using the MassLynx 4.1 software (Waters). Six biological replicates were used for each treatment group. Peak areas, normalized for total ion current for each sample, for individual metabolites were determined and used to calculate fold change after treatment with CHX. All analyzed metabolites are presented in Supplemental Figure 1.

\section{Chromatin immunoprecipitation}

MondoA-V5 was transfected into HeLa cells. Chromatin was cross-linked and sheared as described [19]. Chromatin was incubated overnight with anti-V5 antibody (Thermo Fisher) or mouse IgG (Sigma Aldrich). M-280 sheep anti-mouse Dynabeads (Thermo Fisher) were used to capture and purify immunocomplexes. DNA was purified using a QIAquick PCR Purification Kit (Qiagen) and analyzed using quantitative PCR as described above. Primers were previously described [20].

\section{Promoter activity assay}

TXNIP promoter luciferase assays were performed as described previously [21]. Briefly, cells were transfected with a TXNIP promoter luciferase construct and a CMV-driven $\beta$-galactosidase construct. Following treatments, luciferase and $\beta$-galactosidase activities were determined according to manufacturer's recommendations (Promega, Tropix). Luciferase activity was normalized to $\beta$-galactosidase to control for differences in transfection efficiency.

\section{Fluorescence resonance energy transfer (FRET)}

Widefield microscopy was used to perform live cell imaging on cells expressing Mit-ATEAM as described previously [13]. Briefly, cells transiently transfected with pcDNA3-Mit-ATEAM. Live imaging was conducted using a Nikon A1R with a $\times 40$ lens. Images were captured every hour for $6 \mathrm{~h}$ using the following channels: yellow fluorescent protein (YFP) (excitation $488 \mathrm{~nm}$, emission $525 \mathrm{~nm}$ ), cyan fluorescent protein (CFP) (excitation $405 \mathrm{~nm}$, emission $480 \mathrm{~nm}$ ), and FRET (excitation $405 \mathrm{~nm}$, emission $525 \mathrm{~nm}$ ). The YFP channel was used to designate mitochondria area. The ratio of CFP intensity to FRET intensity was used to determine relative mitochondrial ATP levels.

\section{Immunoblotting}

Immunoblotting was performed as described previously [19]. Primary antibodies were used at a dilution of 1 : 1000 anti-MLXIP/MondoA (Proteintech, 13614-1-AP), 1:2000 anti-VDUP1/TXNIP (MBL, K0205-3), 1:15,000 anti-Tubulin (Molecular Probes, 236-10501), and 1:1000 anti-EIF4E (BioLegend, 693002). Secondary antibodies were used at a dilution of 1:5000 anti-rabbit-HRP (GE Life Sciences, NA-934) or 1:15,000 anti-mouse-HRP (GE Life Sciences, NA-931).

\section{Cell viability assay}

Crystal violet staining was used to determine relative cell viability/proliferation. Cells were stained/fixed using a mixture of $0.05 \%$ crystal violet, $1 \%$ formaldehyde, $1 \%$ methanol, $137 \mathrm{mM} \mathrm{NaCl}, 2.7 \mathrm{mM} \mathrm{KCl}, 10 \mathrm{mM} \mathrm{Na}_{2} \mathrm{HPO}_{4}$, and $1.8 \mathrm{mM} \mathrm{KH}_{2} \mathrm{PO}_{4}$. Following $1 \mathrm{~h}$ in the staining/fixing solution, cells were washed with water until all excess stain was removed, and the plates were dried at room temperature. Crystal violet was extracted from the cells using 1\% SDS and absorbance at $590 \mathrm{~nm}$ was used as a relative measure of total cell numbers.

\section{mRNA sequencing and analysis}

mRNA sequencing was performed as described previously [13]. RNA was harvested using a Quick RNA Miniprep kit (Qiagen), and cDNA libraries were constructed using a stranded mRNA-seq Kit with mRNA Capture Beads (Kapa). The library was sequenced using an Illumina HiSeq 2500. Sequencing was performed by Huntsman Cancer Institute's High Throughput Genomics Core. Reads were aligned to the human genome using STAR. DESeq2 was used to determine differential expression of genes. To determine regulated pathways, we conducted (1) overrepresentation analysis using ConsensusPathDB [22] and (2) gene set enrichment analysis and leading-edge analysis (Broad Institute) [23, 24].

\section{Patient-derived xenograft organoids}

Patient-derived xenograft (PDX) tumors were harvested, processed into organoids (PDxOs) [25-27], and cultured exclusively in a 3D matrigel environment (Corning, growth factor reduced). Fully mature organoids, $>50 \mu \mathrm{m}$ in diameter, were seeded at a density of 50-100 organoids/well in 5\% matrigel, into 384-well tissue cultures 
plates-coated with matrigel to prevent adhesion. Twenty-four hours after seeding, PDxOs were treated with serial dilutions of RocA. We assayed cell viability prior to treatment and after 4 days of treatment with CellTiter-Glo 3D (Promega). Response was determined from technical quadruplicates over three biological replicates.

\section{Statistical methods}

Data represents the mean \pm S.D. for five biological replicates for metabolomics experiments and three biological replicates for all other experiments including RNA-seq. Analysis of variance (ANOVA) was performed to determine significance.

\section{Results}

Translation inhibition drives TXNIP expression

To determine whether TXNIP expression is generally correlated with protein synthesis, we investigated how the expression of a known translation regulator correlates with TXNIP expression. Using the Gene-tissue Expression Database (GTEx) and examining expression in the blood, we identified a strong negative correlation between TXNIP and ribosomal protein L24 (RPL24), which has been shown to correlate well with global changes in protein synthesis in lymphocytes [28,29]. This finding supports the hypothesis that high translation rates suppress TXNIP expression (Fig. 1a).

We next determined whether compounds that block translation at different steps regulate TXNIP expression. We found that treatment of Hela cells with three translation elongation inhibitors, cycloheximide (CHX), emetine, and puromycin (Puro), increased TXNIP expression dramatically (Fig. 1b). Likewise, the translation initiation inhibitor RocA [30] induced TXNIP expression comparably to CHX (Fig. 1c). As expected [6], TXNIP induction by RocA was accompanied by a decrease in glucose uptake (Fig. 1d). siRNA-mediated knockdown of translation

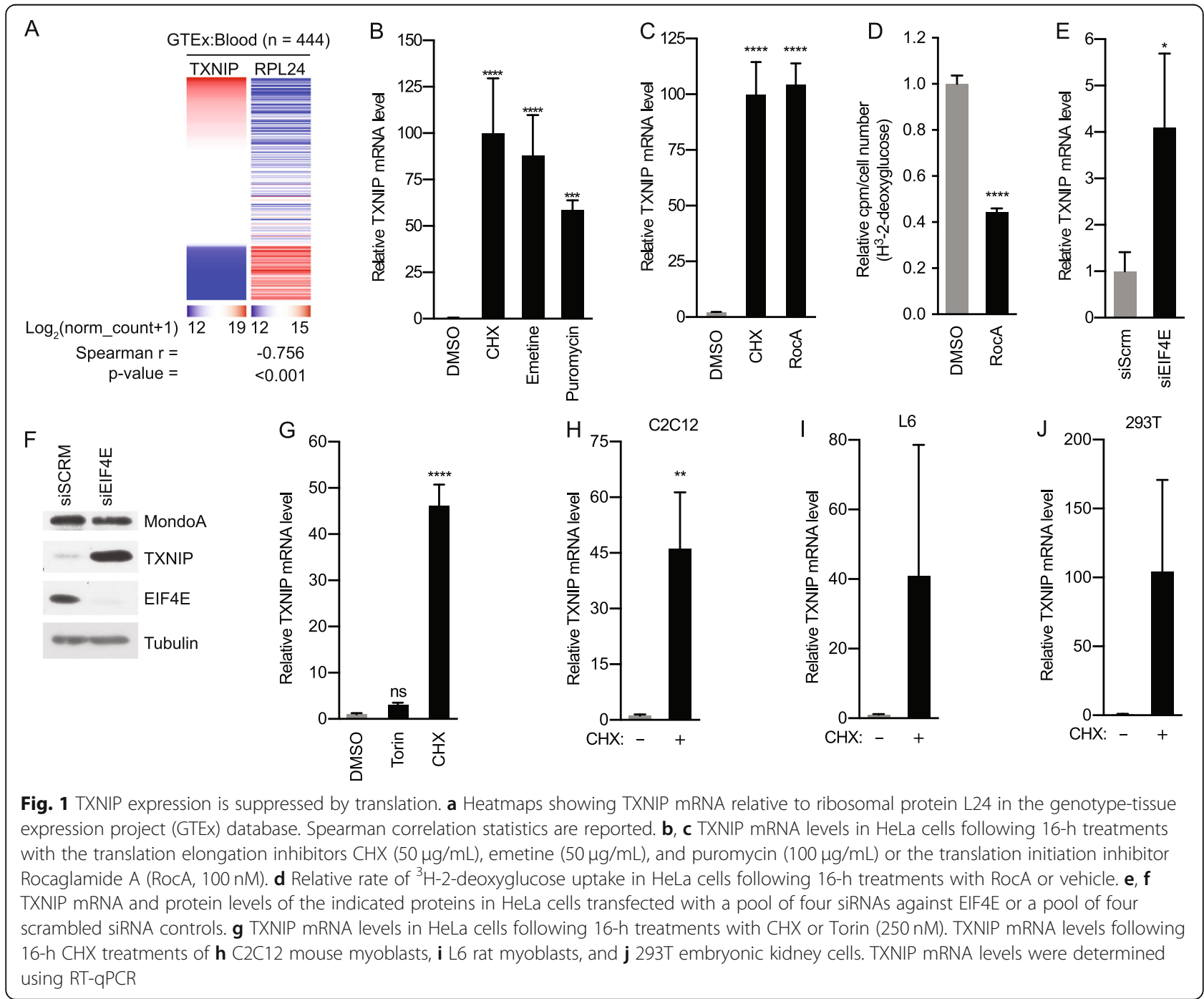


initiation factor EIF4E also increased TXNIP expression (Fig. 1e, f), confirming our findings with the pharmacological inhibitors. It is counter-intuitive that TXNIP protein would accumulate following knockdown of EIF4E; however, TXNIP undergoes both cap-dependent and internal ribosome entry site (IRES)-dependent translation [31]. Therefore, we speculate that IRES-dependent translation accounts for the increase in TXNIP protein levels following EIF4E knockdown. Our previous studies demonstrated that mammalian target of rapamycin complex 1 (mTORC1) suppresses MondoA transcriptional activity and TXNIP expression by competing for its obligate transcriptional partner Mlx [19]. Consistent with our previous findings, the mTORC1 inhibitor Torin increased TXNIP expression, but this increase was much more modest than that observed with CHX (Fig. 1g). This finding suggests that broad translation inhibitors like RocA and CHX increase TXNIP expression by a different mechanism than does Torin, and their action is largely independent of mTORC1. Finally, CHX increased TXNIP expression in C2C12 and L6 myoblasts and HEK293T embryonic kidney cells (Fig. $1 \mathrm{~h}-\mathrm{j})$, suggesting that protein synthesis inhibitors generally increase TXNIP expression. Together, these findings suggest that TXNIP expression, and consequently glucose uptake, is tightly linked to translation rate.

\section{Protein synthesis inhibitors drive MondoA transcriptional activity}

We next evaluated the involvement of MondoA in TXNIP induction in response to protein synthesis inhibition. CHX treatment increased TXNIP expression, in wildtype but not in MondoA ${ }^{-1-}$ mouse embryonic fibroblasts (MEFs) (Fig. 2a). Ectopic expression of MondoA in MondoA ${ }^{-1-}$ MEFs rescued TXNIP induction (Fig. 2b). We tested whether CHX increased MondoA transcriptional activity using several approaches. First, the nuclear localization of MondoA and the amount of MondoA on the TXNIP promoter increased following CHX treatment (Fig. 2c, d). Second, CHX increased the expression from a TXNIP luciferase reporter construct in a manner that was strongly dependent on an intact CACGAG ChoRE about $80 \mathrm{bp}$ upstream of the transcription start site (Fig. 2e). Together these data demonstrate that $\mathrm{CHX}$, and likely other protein synthesis inhibitors, drives MondoA nuclear accumulation, promoter binding, and transcriptional activity.

Because MondoA transcriptional activity is strictly dependent on glucose $[10,11]$, we next determined the requirement for glucose in CHX-driven TXNIP expression. HeLa cells were treated with CHX in DMEM or in glucose-free DMEM. Surprisingly, TXNIP was induced in both media conditions (Fig. 2f), suggesting that $\mathrm{CHX}$ might induce MondoA transcriptional activity independent of glucose. An alternate possibility is that fetal bovine serum (FBS) contains sufficient glucose $(\sim 5 \mathrm{mM})$ such that when present in culture medium at $10 \%$ the resulting concentration of glucose $(\sim 0.5 \mathrm{mM})$ can support MondoA transcriptional activity. To test this hypothesis, we dialyzed FBS to remove small molecules including glucose and then treated cells with $\mathrm{CHX}$ in glucose-free DMEM $+10 \%$ dialyzed FBS. CHX did not increase TXNIP expression in medium containing dialyzed serum; however, adding glucose back to the medium that contained dialyzed serum rescued TXNIP induction (Fig. 2g, h). CHX increased TXNIP expression at all glucose levels tested, and surprisingly decreased the threshold of glucose required for TXNIP induction 5-fold (Fig. 2h). Further, RocA showed glucosedependent changes in TXNIP expression (Supplemental Figure 1A). Thus, glucose is strictly required for $\mathrm{CHX}$ to increase MondoA transcriptional activity and also sensitizes MondoA transcriptional activity to lower glucose levels.

\section{Protein synthesis inhibition drives G6P production}

We next investigated how protein synthesis inhibitors increase MondoA transcriptional activity. We focused on a potential role for mitochondrial function and mtATP for three reasons: (1) protein translation is the most ATP-consuming biosynthetic reaction, (2) MondoA transcriptional activity depends on mtATP [13], (3) higher mtATP levels may sensitize MondoA transcriptional activity and TXNIP expression to lower levels of glucose by increasing levels of G6P [13]. Consistent with a requirement for functional electron transport, inhibition of complex I with metformin completely abrogated TXNIP induction by CHX (Fig. 3a). Likewise, and consistent with a requirement for mtATP, blocking the activity of ATP synthase (complex V) with oligomycin also robustly inhibited TXNIP expression (Fig. 3a). To test the requirement of ATP synthesis further, we used siRNA to deplete ATP5I, which is an essential component of ATP synthase: our previous work established that ATP5I knockdown in HeLa cells blocks the production of mtATP [13]. In this experimental context, ATP5I knockdown reduced background TXNIP expression and completely suppressed its induction by RocA (Fig. 3b). We next determined how protein synthesis inhibition affects mtATP. We expressed a mitochondrial-targeted ATP FRET-biosensor (mitATEAM) in HeLa cells and used live cell imaging to quantify fluorescence [13, 17]. Inhibiting protein synthesis by RocA leads to increased FRET signal indicating accumulation of ATP in the mitochondria (Fig. 3c and Supplemental Figure 1B). These findings suggest a requirement for mtATP synthesis in driving TXNIP expression in response to protein synthesis inhibition. 


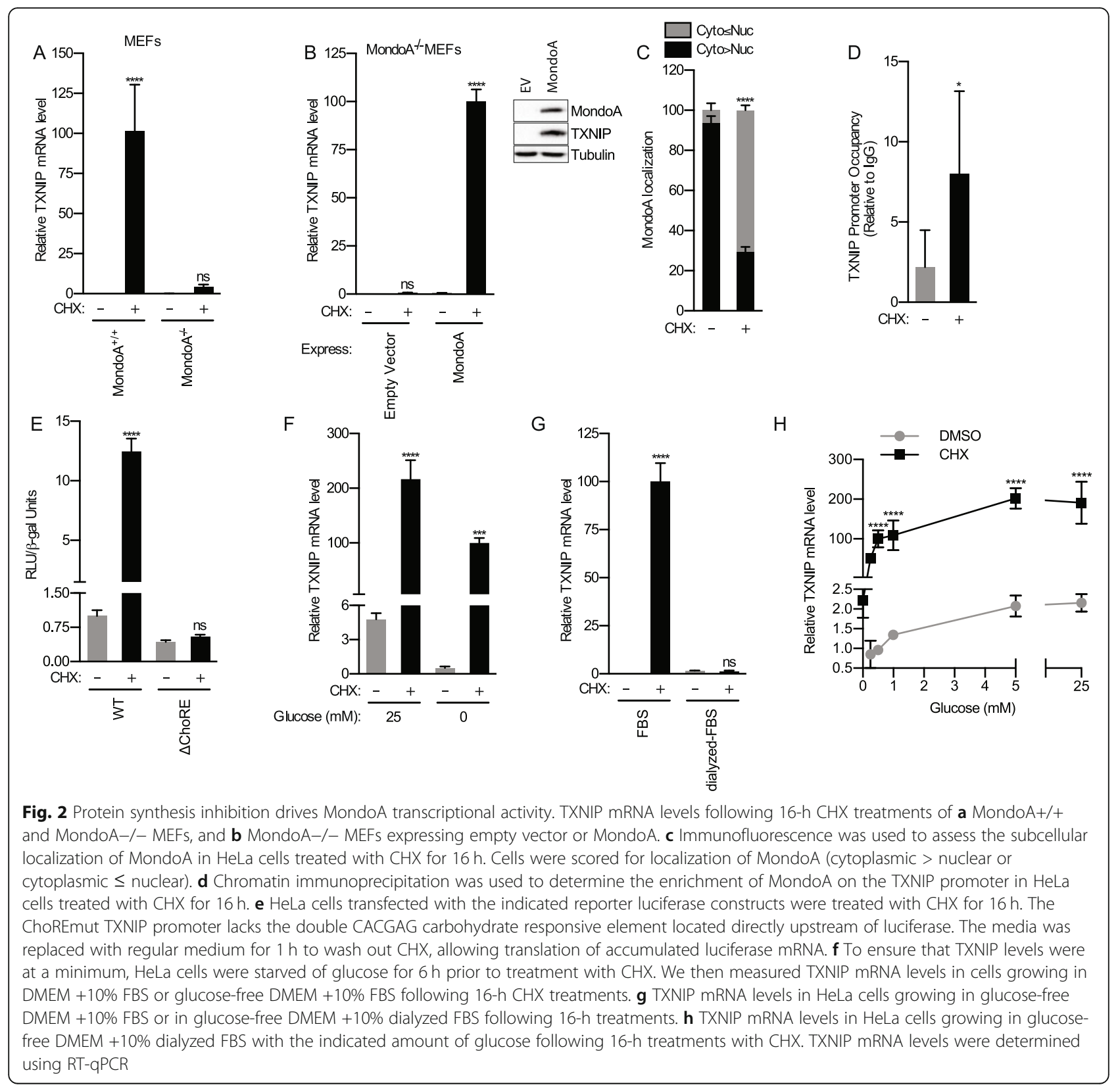

Low $\mathrm{pH}$ medium increases MondoA transcriptional activity by increasing mtATP levels [13]. In that work, we established that mtATP exits the mitochondrial matrix via a channel comprised of the adenine nucleotide transporter (ANT) and the voltage-dependent anion channel (VDAC), where it is used as substrate for VDAC-bound hexokinase II (HKII). Mitochondriabound HKII then transfers a phosphate to cytoplasmic glucose to generate G6P resulting in a stimulation of MondoA transcriptional activity. We tested whether RocA induces TXNIP expression through a similar mechanism in three ways. First, expression of VDAC1(E72Q), which cannot interact with HKII and prevents HKII from interacting with mitochondria [13, 32], blocked the increase in TXNIP expression following RocA treatment (Fig. 3d). By contrast, wildtype VDAC increased TXNIP expression in the presence of RocA. Second, methyl-jasmonate, which removes HKII from the outer membrane of mitochondria [33], blocked RocA induction of TXNIP (Fig. 3e). Third, CHX leads to a dramatic reprogramming of metabolism, including significant changes in the levels of glycolytic and TCA cycle intermediates (Fig. 3f, g and Supplemental Table 1). In particular, G6P levels increased more than 20-fold following CHX treatment (Fig. 3g). Together, these data are consistent with the model that protein synthesis 

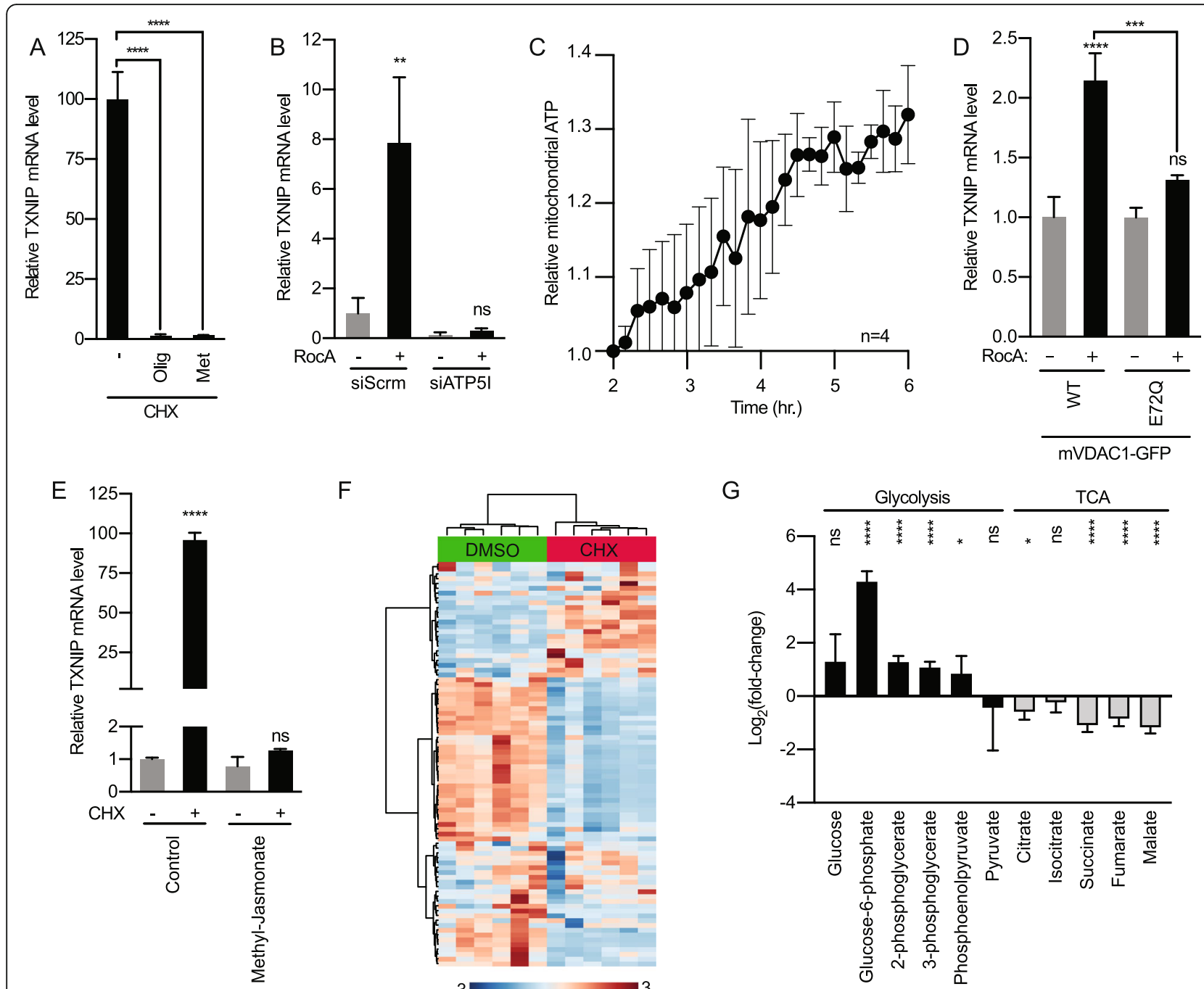

$\mathrm{F}$

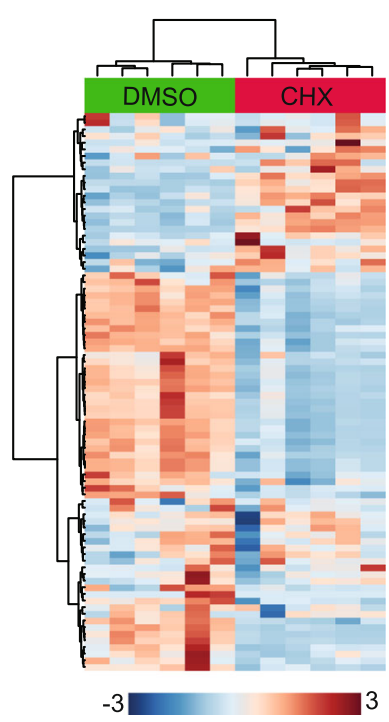

G

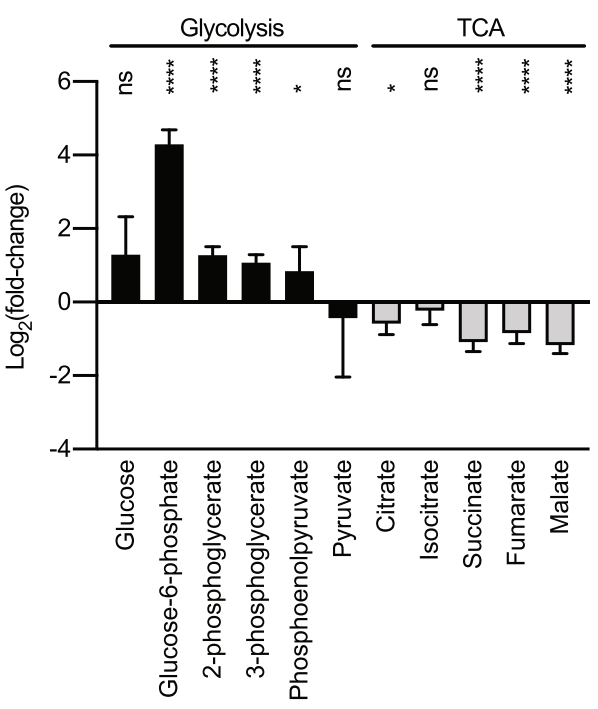

Fig. 3 Protein synthesis inhibition drives G6P synthesis. a TXNIP mRNA levels in HeLa cells treated with CHX and the electron transport chain inhibitor metformin (Met, $5 \mathrm{mM}$ ) or oligomycin (Olig, $1 \mu \mathrm{M}$ ) for $16 \mathrm{~h}$. b TXNIP mRNA levels following a 16-h RocA treatment of HeLa cells transfected with pool of siRNA specific for ATP5I (siATP5I) or a pool of scrambled control siRNAs (siSCRM). c A mitochondrial-targeted ATP FRET biosensor (mitATEAM) was used to determine relative mtATP levels in HeLa cells treated with the protein synthesis inhibitor RocA for up to $6 \mathrm{~h}$. Relative mtATP was determined as the ratio of FRET to CFP intensities. d TXNIP mRNA levels following a 16-h RocA treatment (100 nM) of HeLa cells expressing wildtype mouse VDAC1 (mVDAC1) or VDAC1(E72Q), which cannot bind Hexokinase II. e TXNIP mRNA levels in HeLa cells treated for $16 \mathrm{~h}$ with CHX or methyl-jasmonate $(3 \mathrm{mM})$. $\mathbf{f}$ Heatmap showing relative metabolite levels from HeLa cells treated with $\mathrm{CHX}$ for $16 \mathrm{~h}$. Metabolite levels were assessed through GC-MS. $\mathbf{f} \log _{2}$ (fold-change) of glycolytic and TCA cycle intermediates from HeLa cells treated with CHX for $16 \mathrm{~h}$, relative to control DMSO treatment. TXNIP mRNA levels were determined using RT-qPCR

inhibitors increase mtATP, which is subsequently exported from the mitochondrial matrix through the ANT/VDAC channel, ultimately increasing G6P levels to drive MondoA transcriptional activity.

\section{MondoA and TXNIP are required for the cytotoxic effects of RocA}

Because protein synthesis inhibitors are emerging as potential cancer therapeutics [4-7], we tested whether blocking protein synthesis induced TXNIP expression in cell lines with different oncogenic lesions. $\mathrm{CHX}$ induced
TXNIP in MEFs and in MEFs that expressed an activated allele of HRAS (Fig. 4a) [34]. Further, TXNIP was induced by CHX-treatment in MEFs that lack the TSC2 tumor suppressor and in MDA-MDA-231 cells, which is a triple negative breast cancer (TNBC) cell line that harbors an inactivating mutation in TP53 and activating mutations in KRAS and BRAF (Fig. 4b, c). Further, induction of c-Myc(T58A), which is a stabilized allele of cMyc, did not block TXNIP induction in MDA-MB-231 cells (Fig. 4c). RocA also increased TXNIP protein levels in HeLa cells, MDA-MB-157 cells, which is also a TNBC 


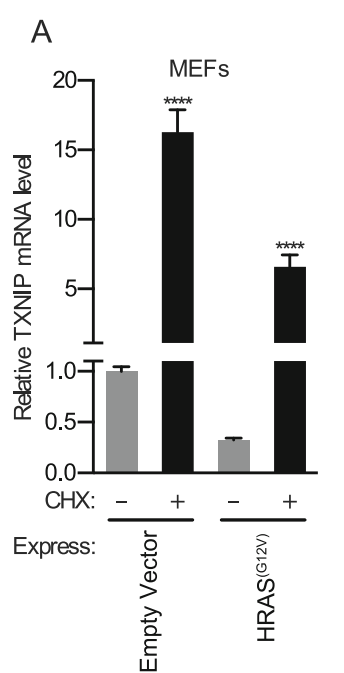

$\mathrm{D}$

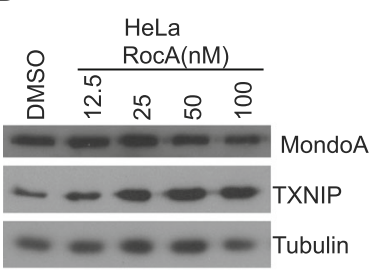

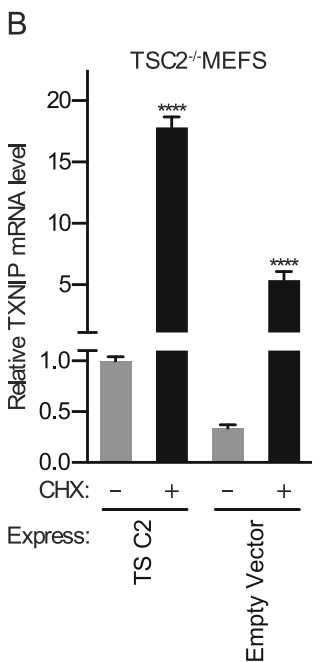

$\mathrm{E}$

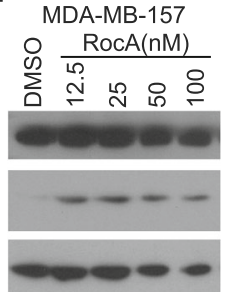

C

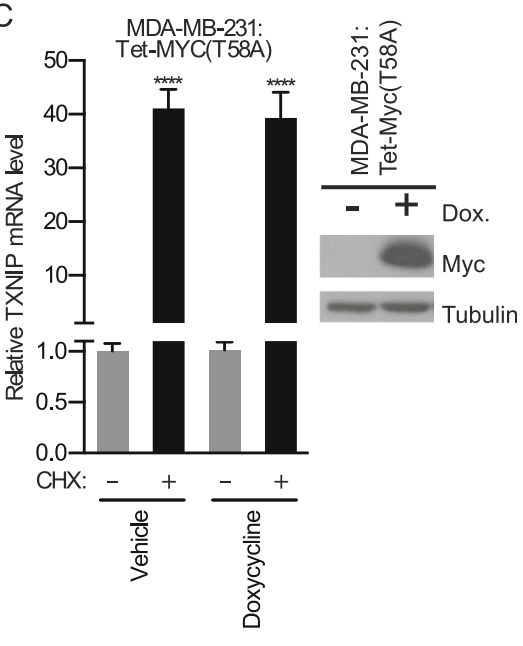

$\mathrm{F}$

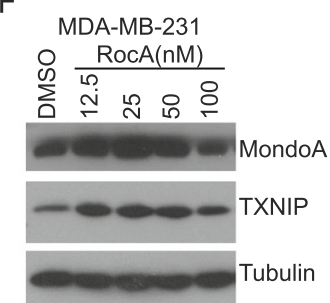

Fig. 4 Protein synthesis inhibition drives TXNIP expression independent of oncogenic burden. TXNIP mRNA levels following a 16-h CHX treatment of a wildtype or HRAS(G12V)-expressing murine embryonic fibroblasts (MEFs), b TSC2-/- MEFs expressing empty vector or humanTSC2, and c MDA-MB-231 expressing tet-inducible MYC(T58A) with or without doxycyline. Immunoblots showing TXNIP, MondoA, and tubulin protein levels following 16-h RocA treatment of $\mathbf{d}$ HeLa cells, e MDA-MB-157 cells, and f MDA-MB-231 cells. TXNIP mRNA levels were determined using RT-qPCR

cell line, and in MBA-MB-231 cells (Fig. 4d, e, f). Together these data demonstrate that RocA can induce TXNIP expression in a variety of cell lines, and its action appears relatively independent of oncogenic burden.

The growth inhibitory effect of RocA has been tested primarily on multiple myeloma cell lines $[6,35]$. Consistent with a potential broad effect of RocA on cell growth, treatment of MDA-MB-157 and MDA-MB-231 breast cancer cells with $100 \mathrm{nM}$ RocA resulted in a timedependent reduction in cell viability such that virtually all the cells were dead after 4 days of treatment (Fig. 5a). We expanded this analysis to 17 organoid cultures derived from breast cancer patients treated at Huntsman Cancer Institute. As with the cell lines, these patientderived xenograft organoids (PDxOs), showed sensitivity to RocA. Ten of 12 estrogen receptor negative (ER-) models were sensitive to RocA, with consistently strong cytotoxicity around $50 \mathrm{nM}$ (Fig. 5b). Most of the estrogen receptor positive (ER+) models, with the exception of HCI-011, were also sensitive to RocA, but sensitivity was attenuated compared to the ER- models: HCI-003 was highly sensitive to RocA like the majority of the ER
- models. Thus, RocA is broadly cytotoxic to breast cancer cells and appears to show preferential killing of cells from ER- breast cancers.

We next determined whether MondoA or TXNIP was required to mediate the cytotoxic effects of RocA. TXNIP-knockout MEFs were less susceptible to RocA than wildtype MEFs (Fig. 5c), consistent with the notion that TXNIP is a RocA effector. Likewise, TXNIP knockdown in MDA-MB-157 cells also partially blocked the cytotoxic effects of RocA (Fig. 5d). Finally, we used CRIS PR-Cas9 editing to generate HeLa cells that lack MondoA (HeLa:MKO) and conducted a RocA dose response experiment. While MondoA-knockout had no effect on cell proliferation in the absence of RocA (Supplemental Figure 1C), we observed that MondoA loss attenuated the cytotoxic effects of RocA and increased the IC50 of RocA from $\sim 15$ to $\sim 25 \mathrm{nM}$ (Fig. 5e). Together these data suggest induction of MondoA transcriptional activity, and the subsequent induction of TXNIP is required for the full cytotoxic effects of RocA. However, the effect of MondoA and TXNIP loss on RocA cytotoxicity, while significant, is subtle suggesting that other pathways must also contribute. 

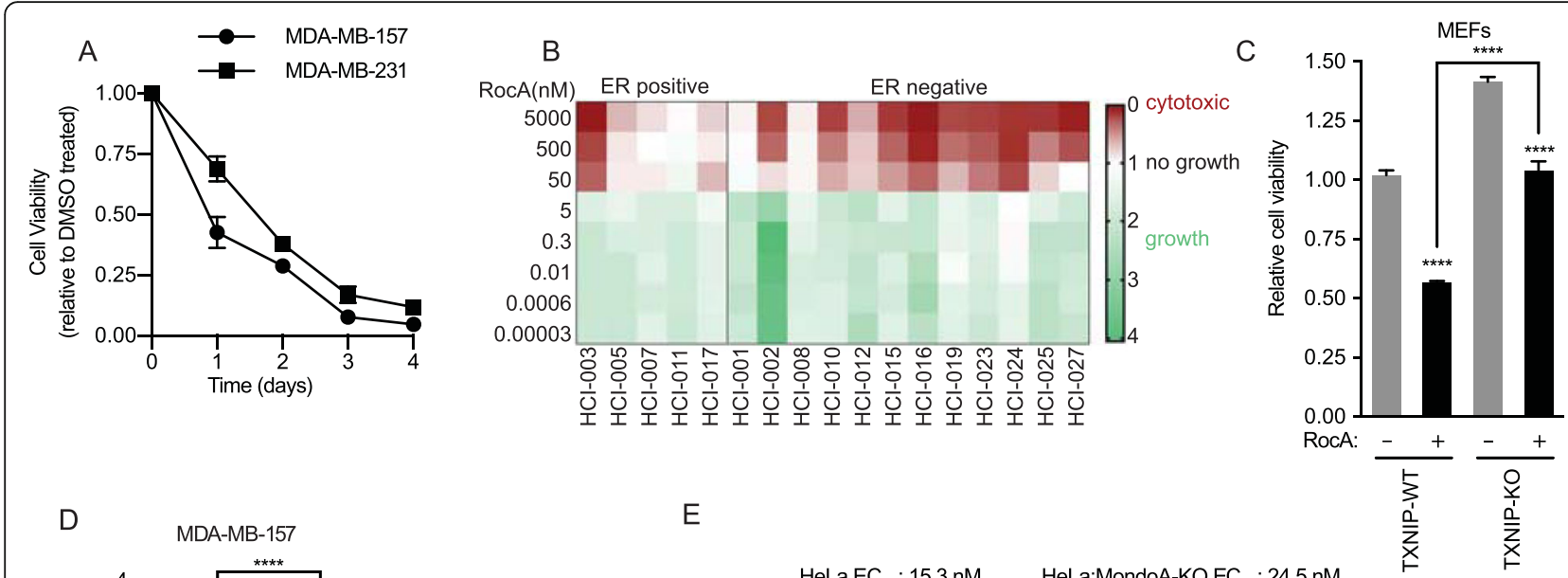

D

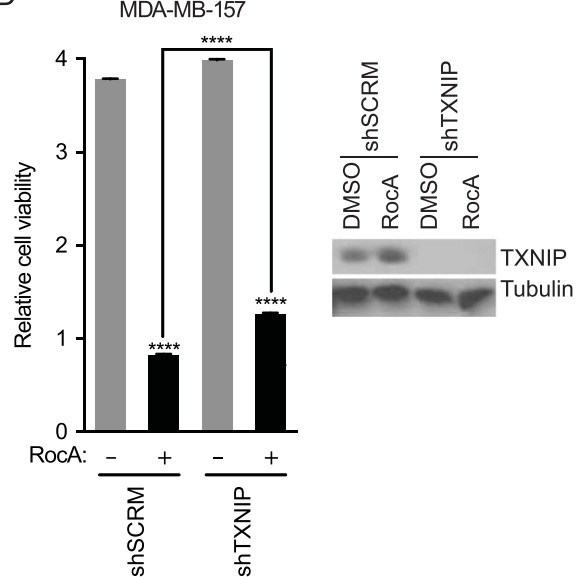

E

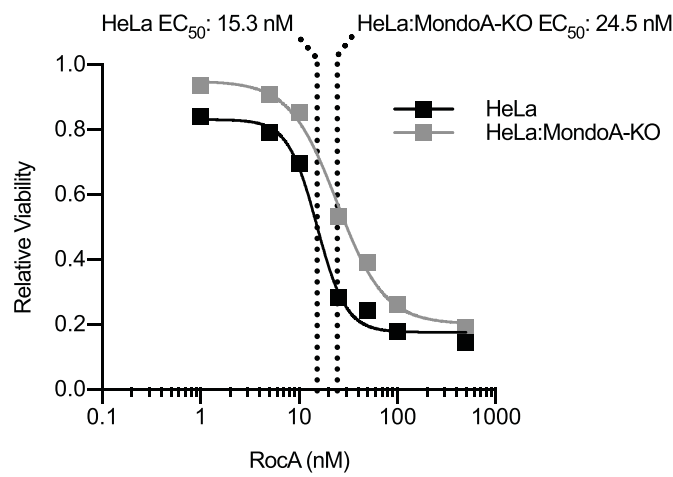

Fig. 5 Cytotoxicity elicited by protein synthesis inhibitors requires TXNIP. a Relative cell viability over the indicated time course of MDA-MB-157 and MDA-MB-231 cells in the presence of RocA (100 nM) was assessed by crystal violet staining. b Viability of patient-derived xenograft organoids (PDxOs) following treatment with RocA at various concentrations. PDxOs are separated into ER+ and ER- groups. c TXNIP+/+ or TXNIP-/- MEFS were treated with RocA for 2 days; then, cell viability was analyzed using crystal violet staining. d MDA-MB-157 cells expressing scrambled shRNA (shScrm) or shTXNIP were treated with 100 nM RocA for 2 days; then, cell viability was analyzed using crystal violet staining. e We previously characterized HeLa cells in which MondoA was knocked out by CRISPR/Cas9 [13]. Cells were treated with RocA for 2 days, and then, cell viability was analyzed using crystal violet staining

\section{The role of MondoA in the transcriptional response to RocA}

To understand the contribution of MondoA to the RocA-dependent transcriptional response, we conducted mRNA-sequencing on RNA prepared from wildtype HeLa or HeLa:MKO cells that had been treated for with $100 \mathrm{nM}$ RocA for $4 \mathrm{~h}$. Using a 2-fold expression change cutoff and a $p$ value of $\leq 0.01$, we identified 1241 genes that were differentially regulated by RocA. Of these, 224 genes were not differentially regulated in the absence of MondoA. This finding suggests that approximately $20 \%$ (224/1241) of the RocA-driven transcriptome requires MondoA (Fig. 6a): both up- and downregulated genes were MondoA-dependent. We next used regression analysis to look for genes that were affected by RocA treatment and genotype. As expected, TXNIP was highly induced by RocA, and its expression was highly dependent on MondoA (Fig. 6a, b). Induction of the TXNIP paralog arrestin domain containing 4 (ARRDC4) by RocA was less robust but was also highly MondoAdependent (Fig. 6a, b). Pathways downregulated following RocA treatment of MondoA knockout cells included extracellular matrix organization and a number of signaling-related pathways (Fig. 6c) [22]. Pathways upregulated following RocA treatment of MondoA knockout cells also included extracellular matrix organization and several pathways involved in sterol biosynthesis. Finally, we conducted gene set enrichment analysis on the differentially regulated genes in HeLa and HeLa:MondoA-KO cells treated with RocA using 13445 pathways in the Molecular Signatures Database [23, 24]. We identified 1033 gene sets that were enriched with a nominal $p$ value of $\leq 0.01$. Leading-edge analysis showed that pathways associated with cell proliferation and cell movement were upregulated, and electron transport and ribosome-related pathways were downregulated in RocA-treated HeLa:MKO cells (Fig. 6d). Together, these data suggest that MondoA is required for the cellular 


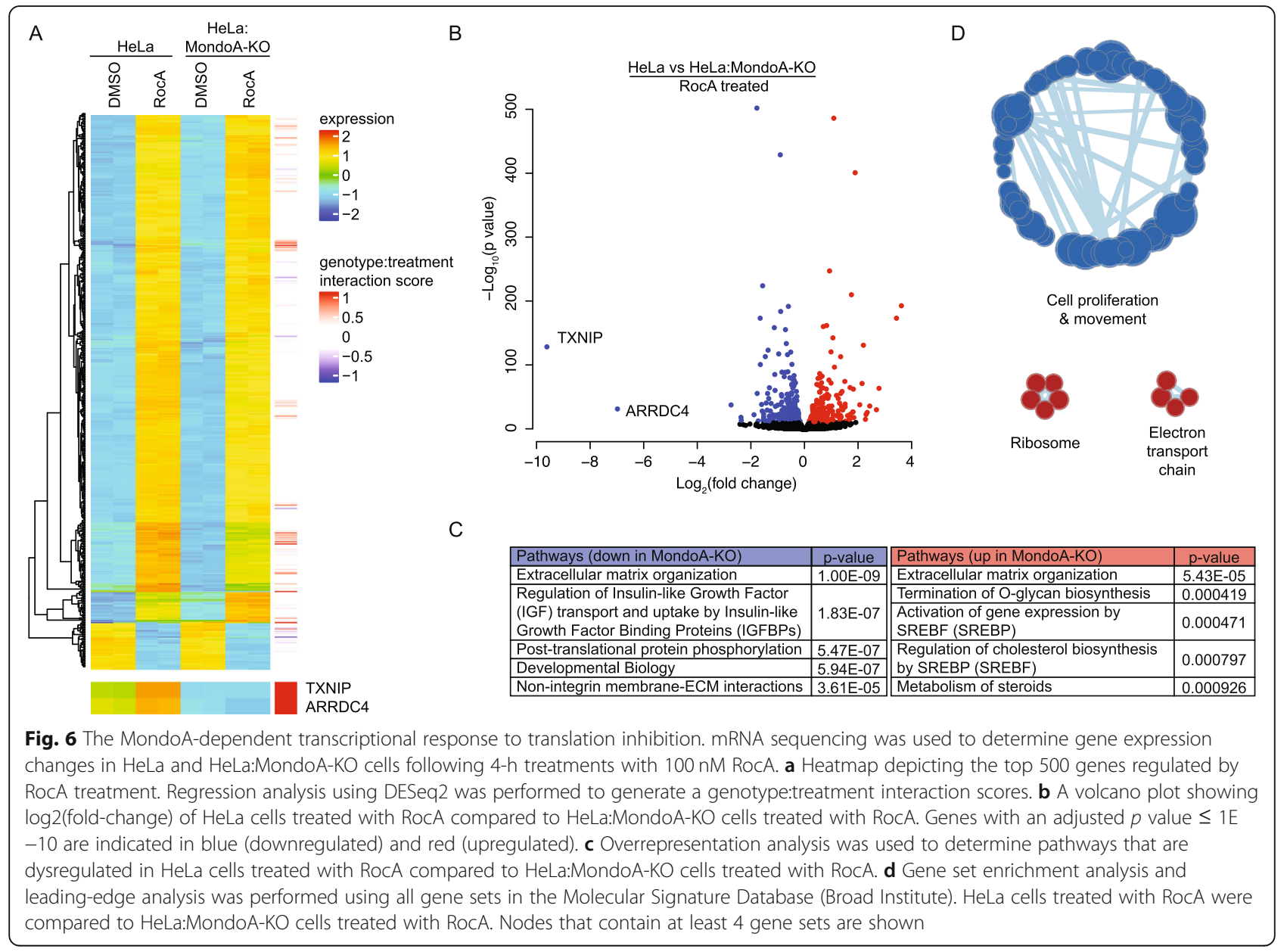

transcriptional response to RocA treatment and may contribute to migratory and growth phenotypes driven by protein synthesis inhibitors.

\section{Discussion}

Translation rate is positively linked to the availability of progrowth signals and the availability of nutrients and charged amino acids [36-38]. A previous report showed that RocA induced TXNIP [6], which correlated with a downregulation of glucose uptake and a blockade of cell growth. Here, we provide a mechanistic framework for this observation, showing that multiple protein synthesis inhibitors, including RocA, drive TXNIP expression by increasing MondoA transcriptional activity. These findings link translation rate to glucose uptake through regulation of MondoA transcriptional activity. We show that protein synthesis inhibitors induce TXNIP expression in a number of different cell lines, apparently independent of oncogenic burden. This finding complements earlier studies showing that a compound related to RocA induces TXNIP expression in a number of cancer cell lines representing a spectrum of malignancies [6].
Cancer cells must coordinate the use and the availability of nutrients to support growth and division. TXNIP is a potent negative regulator of glucose uptake; in fact, its loss or downregulation is sufficient to increase glucose uptake [19, 39], suggesting that low TXNIP levels may be a common route to aerobic glycolysis common in cancer. Consistent with this hypothesis, TXNIP levels are generally lower in tumors compared to normal adjacent tissues [8], and a number of pro-growth/oncogenic pathways suppress TXNIP expression by a variety of mechanisms [19, 20, 34, 40-42]. Together these data suggest that the high demand for ATP driven by translation may result in a reduction of G6P, reduced MondoA transcriptional activity, and low TXNIP expression. Low TXNIP levels increase glucose uptake to help sustain ATP production through glycolysis and potentially replenish stores of glucose-derived amino acids.

TXNIP expression is increased by serum starvation and via inhibition of growth factor signaling pathways $[40,43]$. These treatments also restrict protein translation, raising the possibility that they increase TXNIP expression by altering mATP and/or G6P pools. However, the magnitude of TXNIP induction driven by serum 
starvation or inhibition of growth factor signaling is much less than the increase in TXNIP levels we observe following CHX or RocA treatment. Similarly, we have previously shown that mTORC1 negatively regulates MondoA transcriptional activity and TXNIP expression by binding to MondoA's obligate partner Mlx [19]. Again, the effect of mTORC1 on MondoA activity is relatively subtle compared to effect of $\mathrm{CHX}$ and RocA (Fig. $2 \mathrm{c}$ and g). Together these data suggest that MondoA transcriptional activity is regulated in a relatively tight window under physiological growth conditions, but the supraphysiological levels of mATP and G6P driven by broad inhibition of protein synthesis increases MondoA activity much more potently. Nonetheless, we implicate G6P in activating MondoA transcriptional activity under both physiological conditions and following protein synthesis inhibition. Furthermore, CHX reduces the threshold of glucose needed to drive MondoA transcriptional activity about 5-fold (Fig. 2f). Together these data suggest that inhibition of protein synthesis amplifies the normal regulatory mechanisms that control MondoA transcriptional activity, rather than controlling MondoA activity via an alternate de novo mechanism.

We and others showed previously that MondoA is a critical regulator of glucose-induced transcription [10], which is triggered by G6P. We reported previously that low $\mathrm{pH}(\sim 6.7)$ triggers MondoA transcriptional activity and TXNIP expression [44]. Our recent report demonstrated that low $\mathrm{pH}$ triggers TXNIP expression by increasing mtATP production [13]. Under low $\mathrm{pH}$ conditions mtATP is exported from the mitochondrial matrix, encountering hexokinase II at the outer mitochondrial membrane and generating G6P from cytoplasmic glucose to trigger MondoA transcriptional activity. We show here that inhibition of protein synthesis drives increases MondoA transcriptional activity by a similar mechanism. RocA's induction of MondoA transcriptional activity depends on mtATP synthesis and the interaction of HKII with the outer mitochondrial membrane. Further, our metabolomics experiment showed that CHX treatment results in a dramatic increase in G6P and several other glycolytic intermediates, whereas most TCA intermediates are reduced under these conditions. Together these data suggest that blocking protein synthesis drives MondoA transcriptional activity by increasing mtATP levels, followed by export of mtATP from the mitochondrial matrix and the subsequent increase in G6P: increased G6P triggers MondoA transcriptional activity. It seems most likely that protein synthesis blockade increases mtATP levels by reducing the cytoplasmic demand for ATP. We are currently exploring this and other possibilities.

We showed that both MondoA and TXNIP are partially required for the growth suppressive activity of
RocA, suggesting that the increase in MondoA activity and in TXNIP expression are just not correlated with protein synthesis inhibition, but may be critical for the full therapeutic response to protein synthesis blockade. Our previous work demonstrated that a number of progrowth pathways inhibit MondoA transcriptional activity and TXNIP expression [19, 34, 40, 41], suggesting a potential limitation of protein synthesis inhibitors as cancer therapeutics. However, we show that RocA induced MondoA activity and TXNIP expression in several cell lines independent of oncogenic burden. While our current experiments focus on TXNIP induction by RocA, our previous work demonstrated that a slightly acidic $\mathrm{pH}$ of $\sim 6.7$ drives a gene signature that correlates with good clinical prognosis in breast cancer, and TXNIP is a component of that signature [44]. These findings argue that identifying or developing more specific TXNIP inducers may have therapeutic utility.

In addition to its inhibitory effects on eIF4A, RocA also been shown to disrupt Ras-Raf-MEK signaling. This occurs through the direct binding of prohibitins (PHB1 and PHB2) and their sequestration in the cytosol, which prevents Raf localization to the plasma membrane and its activation by Ras [45]. Given our previous findings that Ras-Raf signaling prevents MondoA transcriptional activity and TXNIP expression [34, 40, 41], it is possible that RocA-driven inhibition of Ras-Raf-MEK signaling also contributes to the increase in MondoA transcriptional activity we observe with RocA treatment.

Finally, MondoA is required for the adaptive transcriptional program driven by RocA and accounts for 20\% of the RocA-induced changes in gene expression. Consistent with our recent demonstration that TXNIP and its paralog ARRDC4 are the principal direct MondoA targets in response to acidosis $[12,13,16]$, their expression is also highly MondoA- and RocA-dependent in these experiments. Leading edge analysis indicates that multiple pathways involved in ribosome function and electron transport chain activity are downregulated in response to RocA, supporting the possibility that translation rate is coupled to mitochondrial function and mtATP levels. Conversely, multiple cell proliferation and migration pathways are upregulated in response to RocA, perhaps reflecting increased mtATP levels. Further experiments will be necessary to fully understand the biological impact the MondoA-dependent changes in gene expression following protein synthesis inhibition.

\section{Conclusions}

Here we show that protein synthesis inhibitors, including specific inhibitors of translation initiation, increase MondoA transcriptional activity resulting in elevated TXNIP expression. Given that TXNIP is a potent negative regulator of glucose uptake, our results suggest 
coordination between translation rate and glucose availability. Mechanistically, inhibition of protein translation increases mtATP, ultimately increasing G6P levels to trigger MondoA transcriptional activity. The MondoATXNIP axis is required for the full cytotoxic effect of RocA, suggesting that negative regulators of MondoA transcriptional activity may limit the efficacy of translational inhibitors in clinical settings. Finally, patientderived organoid models of ER- breast cancers are particularly sensitive to RocA, potentially providing a new therapeutic option against this aggressive and difficultyto-treat breast cancer subtype.

\section{Supplementary Information}

The online version contains supplementary material available at https://doi. org/10.1186/s40170-020-00233-6

Additional file 1: Figure S1. (A) TXNIP mRNA levels following 16-hour $\mathrm{CHX}$ or RocA treatments of HeLa cells in glucose-free or high-glucose media, each with 10\% FBS. TXNIP mRNA levels were determined using RT-qPCR. (B) Widefield confocal images of HeLa cells expressing mit-ATEA $\mathrm{M}$ and treated with RocA for indicated times. The coloring is representative of the ratio of FRET to CFP, where orange/yellow corresponds to higher levels of mtATP. (C) Relative proliferation rate of HeLa and HeLa:MondoA-KO cells over the course of 4 days were analyzed by crystal violet ( $n=3$ for each cell line).

Additional file 2: Table S1.

\section{Abbreviations}

ATP: Adenosine triphosphate; TXNIP: Thioredoxin-interacting protein; RocA: Rocaglamide A; G6P: Glucose 6-phosphate; PDxO: Patient-derived xenograft organoid; mtATP: Mitochondrial ATP; VDAC: Voltage-dependent anion channel; HK: Hexokinase; TNBC: Triple negative breast cancer; ER -: Estrogen receptor negative; ROS: Reactive oxygen species; OMM: Outer mitochondrial membrane; ChoRE: Carbohydrate response element: DMEM: Dulbecco's Minimal Essential Media; FBS: Fetal bovine serum; CHX: Cycloheximide; Puro: Puromycin; GC-MS: Gas chromatograph-mass spectrometry; FRET: Fluorescence resonance energy transfer; YFP: Yellow fluorescent protein; CFP: Cyan fluorescent protein; PDX: Patient-derived xenograft; ANOVA: Analysis of variance; GTEx: Gene-tissue Expression Database; RPL24: Ribosomal protein L24; IRES: Internal ribosome entry site; mTORC1: Mammalian target of rapamycin complex 1; MEFs: Mouse embryonic fibroblasts; ANT: Adenine nucleotide transporter; ER+: Estrogen receptor positive; ARRDC4: Arrestin domain containing 4; Met: Metformin; Olig: Oligomycin

\section{Acknowledgements}

We thank members of the Ayer lab for many helpful discussions during the course of this work. Michelle Mendoza, Assistant Professor in the Department of Oncological Sciences, provided access to the widefield microscope used for the FRET experiments. The sequencing for the RNA-seq experiment was conducted by Huntsman Cancer Institute's High Throughput Genomics Core. The measurement of steady-state metabolites was conducted by the Health Sciences Center at the University of Utah's Metabolomics Core.

\section{Authors' contributions}

DEA, MRK, and BRW conceived the majority of experiments and DEA and BRW wrote the manuscript. BRW conducted all of the experiments presented in the manuscript except for the PDXO experiments. DEA and BEW conceived the PDxO experiments which were conducted by AB and KPG. All authors have approved the manuscript.

\section{Funding}

DEA is supported by R01CA222650, funds from the Huntsman Cancer Foundation, and the HCl Cancer Center Support Grant P30CA042014-31. BEW is supported by U54 CA224076

\section{Availability of data and materials}

RNA-seq data is available at GEO under the accession number GSE153499. The metabolomics dataset is available in Supplemental Table 1. All of the remaining data generated or analyzed during this study are included in this published article and its supplementary information files.

\section{Ethics approval and consent to participate}

De-identified breast tumor tissues were collected by the Huntsman Cancer Institute Tissue Resource and Applications Core Facility with informed consent from patients at the Huntsman Cancer Hospital and the University of Utah Hospitals and Clinics under a protocol approved by the University of Utah Institutional Review Board [25].

\section{Consent for publication}

Our manuscript contains no data from any identifiable individuals.

\section{Competing interests}

The authors declare that they have no competing interests.

\section{Author details}

${ }^{1}$ Department of Oncological Sciences, Huntsman Cancer Institute, University of Utah, Salt Lake City, UT 84112, USA. ${ }^{2}$ Present Address: Department of Biological Chemistry, David Geffen School of Medicine, University of California, Los Angeles, Los Angeles, CA 90095, USA. ${ }^{3}$ Present Address: Translational Genomics Research Institute, Phoenix, AZ 85004, USA.

${ }^{4}$ Department of Surgery, Huntsman Cancer Institute, University of Utah, Salt Lake City, UT 84112, USA.

Received: 1 July 2020 Accepted: 25 November 2020

Published online: 04 December 2020

\section{References}

1. Hsieh AL, Dang CV. MYC, Metabolic synthetic lethality, and cancer. Recent Results Cancer Res. 2016:207:73-91.

2. Lettieri-Barbato D, Aquilano K. Pushing the limits of cancer therapy: the nutrient game. Front Oncol. 2018;8:148.

3. Wellen KE, Thompson CB. Cellular metabolic stress: considering how cells respond to nutrient excess. Molecular cell. 2010;40(2):323-32.

4. Bhat M, Robichaud N, Hulea L, Sonenberg N, Pelletier J, Topisirovic I. Targeting the translation machinery in cancer. Nat Rev Drug Discov. 2015; 14(4):261-78.

5. Martineau Y, Muller D, Pyronnet S. Targeting protein synthesis in cancer cells. Oncoscience. 2014;1(7):484-5.

6. Santagata S, Mendillo ML, Tang YC, Subramanian A, Perley CC, Roche SP, et al. Tight coordination of protein translation and HSF1 activation supports the anabolic malignant state. Science. 2013;341(6143):1238303.

7. Truitt ML, Ruggero D. New frontiers in translational control of the cancer genome. Nat Rev Cancer. 2017:17(5):332.

8. O'Shea JM, Ayer DE. Coordination of nutrient availability and utilization by MAX- and MLX-centered transcription networks. Cold Spring Harbor Perspect Med. 2013;3(9):a014258.

9. Shalev A. Minireview: Thioredoxin-interacting protein: regulation and function in the pancreatic beta-cell. Mol Endocrinol. 2014;28(8):1211-20.

10. Stoltzman CA, Peterson CW, Breen KT, Muoio DM, Billin AN, Ayer DE. Glucose sensing by MondoA:Mlx complexes: a role for hexokinases and direct regulation of thioredoxin-interacting protein expression. Proc Nat Acad Sci U S A. 2008:105(19):6912-7.

11. Peterson CW, Stoltzman CA, Sighinolfi MP, Han KS, Ayer DE. Glucose controls nuclear accumulation, promoter binding, and transcriptional activity of the MondoA-Mlx heterodimer. Mol Cell Biol. 2010;30(12):2887-95.

12. Sans CL, Satterwhite DJ, Stoltzman CA, Breen KT, Ayer DE. MondoA-Mlx heterodimers are candidate sensors of cellular energy status: mitochondrial localization and direct regulation of glycolysis. Mol Cell Biol. 2006;26(13): 4863-71. 
13. Wilde BR, Ye Z, Lim TY, Ayer DE. Cellular acidosis triggers human MondoA transcriptional activity by driving mitochondrial ATP production. Elife. 2019; 8:e40199.

14. Minn AH, Hafele C, Shalev A. Thioredoxin-interacting protein is stimulated by glucose through a carbohydrate response element and induces beta-cell apoptosis. Endocrinology. 2005;146(5):2397-405.

15. Wilde BR, Ayer DE. Interactions between Myc and MondoA transcription factors in metabolism and tumourigenesis. Br J Cancer. 2015;113(11):1529-33.

16. Stoltzman CA, Kaadige MR, Peterson CW, Ayer DE. MondoA senses nonglucose sugars: regulation of thioredoxin-interacting protein (TXNIP) and the hexose transport curb. J Biol Chem. 2011;286(44):38027-34.

17. Imamura H, Nhat KP, Togawa H, Saito K, lino R, Kato-Yamada $Y$, et al. Visualization of ATP levels inside single living cells with fluorescence resonance energy transfer-based genetically encoded indicators. Proc Natl Acad Sci U S A. 2009:106(37):15651-6.

18. Heidelberger C, Chaudhuri NK, Danneberg P, Mooren D, Griesbach L, Duschinsky $R$, et al. Fluorinated pyrimidines, a new class of tumourinhibitory compounds. Nature. 1957;179(4561):663-6.

19. Kaadige MR, Yang J, Wilde BR, Ayer DE. MondoA-Mlx transcriptional activity is limited by mTOR-MondoA interaction. Mol Cell Biol. 2015;35(1):101-10.

20. Shen L, O'Shea JM, Kaadige MR, Cunha S, Wilde BR, Cohen AL, et al. Metabolic reprogramming in triple-negative breast cancer through Myc suppression of TXNIP. Proc Natl Acad Sci U S A. 2015;112(17):5425-30.

21. Billin AN, Eilers AL, Queva C, Ayer DE. Mlx, a novel Max-like BHLHZip protein that interacts with the Max network of transcription factors. J Biol Chem. 1999:274(51):36344-50

22. Kamburov A, Stelzl U, Lehrach $H$, Herwig R. The ConsensusPathDB interaction database: 2013 update. Nucleic Acids Res. 2013:41(Database issue):D793-800

23. Mootha VK, Lindgren CM, Eriksson KF, Subramanian A, Sihag S, Lehar J, et al. PGC-1alpha-responsive genes involved in oxidative phosphorylation are coordinately downregulated in human diabetes. Nat Genet. 2003;34(3):267-73.

24. Subramanian A, Tamayo P, Mootha VK, Mukherjee S, Ebert BL, Gillette MA, et al. Gene set enrichment analysis: a knowledge-based approach for interpreting genome-wide expression profiles. Proc Natl Acad Sci U S A. 2005;102(43):15545-50

25. DeRose YS, Gligorich KM, Wang G, Georgelas A, Bowman P, Courdy SJ, et al. Patient-derived models of human breast cancer: protocols for in vitro and in vivo applications in tumor biology and translational medicine. Curr Protoc Pharmacol. 2013;14(Unit14):23.

26. DeRose YS, Wang G, Lin YC, Bernard PS, Buys SS, Ebbert MT, et al. Tumor grafts derived from women with breast cancer authentically reflect tumor pathology, growth, metastasis and disease outcomes. Nat Med. 2011;17(11): 1514-20.

27. Gligorich KM, Vaden RM, Shelton DN, Wang G, Matsen CB, Looper RE, et al. Development of a screen to identify selective small molecules active against patient-derived metastatic and chemoresistant breast cancer cells. Breast Cancer Res. 2013;15(4):R58

28. Barna M, Pusic A, Zollo O, Costa M, Kondrashov N, Rego E, et al. Suppression of Myc oncogenic activity by ribosomal protein haploinsufficiency. Nature. 2008:456(7224):971-5.

29. Signer RA, Magee JA, Salic A, Morrison SJ. Haematopoietic stem cells require a highly regulated protein synthesis rate. Nature. 2014;509(7498):49-54

30. Iwasaki S, Floor SN, Ingolia NT. Rocaglates convert DEAD-box protein elF4A into a sequence-selective translational repressor. Nature. 2016:534(7608): 558-61.

31. Lampe S, Kunze M, Scholz A, Brauss TF, Winslow S, Simm S, et al. Identification of the TXNIP IRES and characterization of the impact of regulatory IRES trans-acting factors. Biochim Biophys Acta Gene Regul Mech. 2018;1861(2):147-57.

32. Zaid H, Abu-Hamad S, Israelson A, Nathan I, Shoshan-Barmatz V. The voltage-dependent anion channel-1 modulates apoptotic cell death. Cell Death Differ. 2005;12(7):751-60

33. Goldin N, Arzoine L, Heyfets A, Israelson A, Zaslavsky Z, Bravman T, et al. Methyl jasmonate binds to and detaches mitochondria-bound hexokinase. Oncogene. 2008;27(34):4636-43.

34. Ye Z, Ayer DE. Ras suppresses TXNIP expression by restricting ribosome translocation. Mol Cell Biol. 2018:38:20.

35. Wu Y, Giaisi M, Kohler R, Chen WM, Krammer PH, Li-Weber M. Rocaglamide breaks TRAIL-resistance in human multiple myeloma and acute T-cell leukemia in vivo in a mouse xenogtraft model. Cancer Lett. 2017;389:70-7.
36. Condon KJ, Sabatini DM. Nutrient regulation of mTORC1 at a glance. J Cell Sci. 2019;132:21

37. Kimball SR, Jefferson LS. Control of protein synthesis by amino acid availability. Curr Opin Clin Nutr Metab Care. 2002:5(1):63-7.

38. Wolfson RL, Sabatini DM. The dawn of the age of amino acid sensors for the mTORC1 pathway. Cell Metab. 2017;26(2):301-9.

39. Hui ST, Andres AM, Miller AK, Spann NJ, Potter DW, Post NM, et al. Txnip balances metabolic and growth signaling via PTEN disulfide reduction. Proc Natl Acad Sci U S A. 2008:105(10):3921-6.

40. Elgort MG, O'Shea JM, Jiang Y, Ayer DE. Transcriptional and translational downregulation of thioredoxin interacting protein is required for metabolic reprogramming during G(1). Genes Cancer. 2010;1(9):893-907.

41. Parmenter TJ, Kleinschmidt M, Kinross KM, Bond ST, Li J, Kaadige MR, et al. Response of BRAF-mutant melanoma to BRAF inhibition is mediated by a network of transcriptional regulators of glycolysis. Cancer Discov. 2014;4(4): 423-33.

42. Waldhart AN, Dykstra H, Peck AS, Boguslawski EA, Madaj ZB, Wen J, et al Phosphorylation of TXNIP by AKT mediates acute influx of glucose in response to insulin. Cell Rep. 2017;19(10):2005-13.

43. Terragni J, Graham JR, Adams KW, Schaffer ME, Tullai JW, Cooper GM Phosphatidylinositol 3-kinase signaling in proliferating cells maintains an anti-apoptotic transcriptional program mediated by inhibition of FOXO and non-canonical activation of NFkappaB transcription factors. BMC Cell Biol. 2008;9:6.

44. Chen JL, Merl D, Peterson CW, Wu J, Liu PY, Yin H, et al. Lactic acidosis triggers starvation response with paradoxical induction of TXNIP through MondoA. PLoS Genet. 2010;6(9):e1001093.

45. Polier G, Neumann J, Thuaud F, Ribeiro N, Gelhaus C, Schmidt H, et al. The natural anticancer compounds rocaglamides inhibit the Raf-MEK-ERK pathway by targeting prohibitin 1 and 2. Chem Biol. 2012;19(9):1093-104

\section{Publisher's Note}

Springer Nature remains neutral with regard to jurisdictional claims in published maps and institutional affiliations.
Ready to submit your research? Choose BMC and benefit from:

- fast, convenient online submission

- thorough peer review by experienced researchers in your field

- rapid publication on acceptance

- support for research data, including large and complex data types

- gold Open Access which fosters wider collaboration and increased citations

- maximum visibility for your research: over $100 \mathrm{M}$ website views per year

At BMC, research is always in progress.

Learn more biomedcentral.com/submissions 